Pacific Journal of Mathematics

ADJOINT BOUNDARY VALUE PROBLEMS FOR
COMPACTIFIED SINGULAR DIFFERENTIAL OPERATORS 


\title{
ADJOINT BOUNDARY VALUE PROBLEMS FOR COMPACTIFIED SINGULAR DIFFERENTIAL OPERATORS
}

\author{
PHILIP W. WALKER
}

This paper is concerned with differential operators and their adjoints induced in the Hilbert space $\mathscr{L}^{2}(w)$ by an operator $(1 / w) l$ where $l$ is an $n$th order singular differential operator and $w$ is a weight. It is shown that weights may be chosen and boundary conditions may be imposed so that the structure of these operators is similar to that of regular differential operators.

1. Preliminaries. Throughout $l$ will denote an operator of the form,

$$
l(y)=y^{(n)}+\sum_{k=1}^{n} p_{k} y^{(n-k)},
$$

where each $p_{k}$ is an $(n-k)$ times continuously differentiable complex valued function on an interval $(a, b)$. We allow $a=-\infty$ and/or $b=$ $\infty$. The formal adjoint of $l$ will be denoted by $l^{+}$. Hence

$$
l^{+}(y)=(-1)^{n} y^{(n)}+\sum_{k=1}^{n}(-1)^{n-k}\left(\bar{p}_{k} y\right)^{(n-k)}
$$

for all $n$ times differentiable $y$ on $(a, b)$.

If $y$ is an $(n-1)$ times differentiable function then $k(y)$ will denote the vector valued function, column $\left(y, y^{\prime}, \cdots, y^{(n-1)}\right)$, and if each of $y_{1}, y_{2}, \cdots, y_{n}$ is an $(n-1)$ times differentiable function then $\boldsymbol{K}\left(y_{1}\right.$, $\left.\cdots, y_{n}\right)$ will denote the matrix valued function whose $(i, j)$ entry is $y_{j}^{(i-1)}$ for $1 \leqq i, j \leqq n$.

$\mathscr{C}$ will denote the complex numbers, the space of all complex $n \times 1$ column vectors will be denoted by $\mathscr{C}^{n}$, and the space of all complex $n \times n$ matrices will be denoted by $\mathscr{C}^{n}$. If $M$ is a matrix then $M^{*}$ will denote its conjugate transpose.

Definition 1.1. Let $\left(\varphi_{1}, \cdots, \varphi_{n}\right)$ be a sequence of linearly independent solutions to

$$
l(y)=0 \quad \text { on } \quad(a, b) .
$$

The statement that $\left(\theta_{1}, \cdots, \theta_{n}\right)$ is the adjoint of $\left(\varphi_{1}, \cdots, \varphi_{n}\right)$ means that $\theta_{k}$ is the complex conjugate of the $(k, n)$ entry of the matrix $\left[K\left(\varphi_{1}, \cdots, \varphi_{n}\right)\right]^{-1}$ for $k=1,2, \cdots, n$.

We shall make use of the following facts concerning adjoints of fundamental systems of solutions to Eq. (1.2). 
LEMMA 1.2. Let $\left(\theta_{1}, \cdots, \theta_{n}\right)$ be the adjoint of $\left(\varphi_{1}, \cdots, \varphi_{n}\right), a$ sequence of linearly independent solutions of Eq. (1.2). Let $t_{0} \in(a, b)$ and let $f:(a, b) \rightarrow \mathscr{C}$ be Lebesgue integrable on every compact subinterval of $(a, b)$. It follows that

$$
l(y)=f \text { a.e. on }(a, b)
$$

if and only if

$$
\begin{aligned}
\boldsymbol{k}(y)(t)= & \boldsymbol{K}\left(\varphi_{1}, \cdots, \varphi_{n}\right)(t)\left\{\left[\boldsymbol{K}\left(\varphi_{1}, \cdots, \varphi_{n}\right)\left(t_{0}\right)\right]^{-1} \boldsymbol{k}(y)\left(t_{0}\right)\right. \\
& \left.+\int_{t_{0}}^{t}\left[\left(\theta_{1}, \theta_{2}, \cdots, \theta_{n}\right)(s)\right]^{*} f(s) d s\right\}
\end{aligned}
$$

for all $t$ in $(a, b)$.

This follows from consideration of the standard vector matrix formulation of Eq. (1.3) and from Eq. (3.2), p. 75 of [1].

LEMMA 1.3. Let $\varphi_{k}$ and $\theta_{k}, k=1, \cdots, n$, be as in Lemma 1.2. It follows that $\left(\theta_{1}, \cdots, \theta_{n}\right)$ is a linearly independent sequence of solutions to

$$
l^{+}(y)=0 \quad \text { on }(a, b) .
$$

See problem 19, p. 101 of [1] and Theorem 5, p. 38 of [5]. Note that in the latter reference the formal adjoint differential operator is defined without taking complex conjugates. The same is true in Ref. [2] wherein on p. 69 in Corollary 3.8.2c we find justification for

Lemma 1.4. Let $\varphi_{k}$ and $\theta_{k}$ be as in Lemma 1.2. Then

$$
\left[\boldsymbol{K}\left(\theta_{1}, \cdots, \theta_{n}\right)\right]^{*} \boldsymbol{P}\left[\boldsymbol{K}\left(\varphi_{1}, \cdots, \varphi_{n}\right)\right] \equiv \boldsymbol{I} \text { on }(a, b) \text {. }
$$

Where $\boldsymbol{I}$ is the $n \times n$ identity matrix and $\boldsymbol{P}$ is the concomitant matrix of $l$.

See $\S 3.7$ of [2] or p. 285 of [1] (therein denoted $B$ ) for the definition of $\boldsymbol{P}$.

By a weight we mean a positive real valued continuous function. If $w$ is a weight on $(a, b)$ then $\mathscr{L}^{2}(w)$ will denote the Hilbert space of all (equivalence classes of) Lebesgue measurable $f:(a, b) \rightarrow \mathscr{C}$ satisfying $\int_{a}^{b}|f|^{2} w<\infty$. If $f, g \in \mathscr{L}^{2}(w)$ then $\langle f, g\rangle=\int_{a}^{b} f \bar{g} w$, and $\|f\|=\sqrt{\langle f, f\rangle}$.

Definition 1.5. The statement that $w$ is a compactifying weight 
for $l$ means that all solutions to Eq. (1.2) and the all solutions to Eq. (1.5) lie in $\mathscr{L}^{2}(w)$.

Since the solution spaces of Eqs. (1.2) and (1.3) are finite dimensional spaces of continuous functions it follows that every operator $l$ has many compactifying weights. The reason for the terminology is that operators induced in $\mathscr{L}^{2}(w)$ by $(1 / w) l$ and certain boundary conditions will have compact inverses.

The study of operators with a compactifying weight is in some sense complementary to the study of those with an $l$-admissible weight considered in [7].

2. Solutions of the eigenvalue equation. Our first theorem shows that solutions to differential equations with a compactifying weight behave in a manner similar to solutions of second order selfadjoint equations of the limit-circle type. (See $\S 2$ p. 225 of [1].)

THEOREM 2.1. Let $w$ be a compactifying weight for $l$. If $f \in$ $\mathscr{L}^{2}(w)$ and $\lambda \in \mathscr{C}$ ( $\gamma$ may be real, even zero) then every solution to

$$
l(y)=w(\lambda y+f) \text { a.e. on }(a, b)
$$

lies in $\mathscr{L}^{2}(w)$.

Proof. Suppose that $y$ satisfies Eq. (2.1). Let $t_{0} \in(a, b)$ and let $\left(\varphi_{1}, \cdots, \varphi_{n}\right)$ and $\left(\theta_{1}, \cdots, \theta_{n}\right)$ be as in Lemma 1.2. Inspection of the first components of vector Eq. (1.4) shows that

$$
y(t)=\varphi(t)+\sum_{k=1}^{n} \varphi_{k}(t) \int_{t_{0}}^{t} \overline{\theta_{k}(s)}(f(s)+\lambda y(s)) w(s) d s
$$

for all $t \in(\mathrm{a}, b)$ where $\varphi$ is a solution to Eq. (1.2). Thus for $t_{0} \leqq t<b$ it follows from the Cauchy-Schwartz inequality that

$$
\begin{aligned}
|y(t)| \leqq & |\varphi(t)|+\sum_{k=1}^{n}\left|\varphi_{k}(t)\right|\left\{\left\|\theta_{k}\right\| \cdot\|f\|\right. \\
& \left.+|\lambda|\left(\int_{t_{0}}^{t}\left|\theta_{k}(s)\right|^{2} w(s) d s\right)^{1 / 2}\left(\int_{t_{0}}^{t}|y(s)|^{2} w(s) d s\right)^{1 / 2}\right\} .
\end{aligned}
$$

Thus

$$
|y(t)| \leqq|u(t)|+g(t)\left\{\int_{t_{0}}^{t}|y(s)|^{2} w(s) d s\right\}^{1 / 2}
$$

for $t_{0} \leqq t<b$ where $u=|\varphi|+\sum_{k=1}^{n}\left|\varphi_{k}\right| \cdot\left\|\theta_{k}\right\| \cdot\|f\|$ and

$$
g=\sum_{k=1}^{n}\left|\varphi_{k}\right| \cdot|\lambda| \cdot \| \theta_{k}|| \text {. }
$$

Note that each of $u$ and $g$ is in $\mathscr{L}^{2}(w)$. Applying Theorem 1 of [4] 
with $G(t)=t^{2}$, and $\alpha(t)=\beta(t) \equiv 1 / 2$, we have that

$$
\int_{t_{0}}^{t}|y(s)|^{2} w(s) d s \leqq c \int_{t_{0}}^{t}|u(s)|^{2} w(s) d s
$$

for $t_{0} \leqq t<b$ where $c=2 \exp (2\|g\|)$. A similar argument shows that for $a<\tau \leqq t_{0}$,

$$
\int_{\tau}^{t_{0}}|y(s)|^{2} w(s) d s \leqq c \int_{\tau}^{t_{0}}|u(s)|^{2} w(s) d s .
$$

Thus $y \in \mathscr{L}^{2}(w)$.

The next theorem provides a method for specifying initial conditions for the solutions of Eq. (2.1) at the endpoints of the interval $(a, b)$.

THEOREM 2.2. Let $w$ be a compactifying weight for $l$, let $f \in \mathscr{L}^{2}(w)$ and let $\lambda \in \mathscr{C}$. Let $\left(\varphi_{1}, \cdots, \varphi_{n}\right)$ be a linearly independent sequence of solutions of $E q .(1.2)$ and let $\boldsymbol{Y}=\boldsymbol{K}\left(\Phi_{1}, \cdots, \Phi_{n}\right)$. If $y$ is a solution to $E q$. (2.1) then

$$
\lim _{t \rightarrow a} \boldsymbol{Y}^{-1}(t) \boldsymbol{k}(y)(t) \quad \text { and } \quad \lim _{t \rightarrow b} \boldsymbol{Y}^{-1}(t) \boldsymbol{k}(y)(t)
$$

exist and are finite. Moreover, if $c \in \mathscr{C}^{n}$ then there is exactly one solution $y$ of Eq. (2.1) satisfying

$$
\lim _{t \rightarrow a} \boldsymbol{Y}^{-1}(t) \boldsymbol{k}(y)(t)=\boldsymbol{c},
$$

and there is exactly one solution $y$ of Eq. (2.1) satisfying

$$
\lim _{t \rightarrow b} \boldsymbol{Y}^{-1}(t) \boldsymbol{k}(y)(t)=\boldsymbol{c} .
$$

Proof. Let $\left(\theta_{1}, \cdots, \theta_{n}\right)$ be the adjoint of $\left(\varphi_{1}, \cdots, \varphi_{n}\right)$ and let $t_{0} \in$ $(a, b)$. From Eq. (1.4) it follows that if $y$ satisfies Eq. (2.1) then

$$
\begin{aligned}
\boldsymbol{Y}^{-1}(t) \boldsymbol{k}(y)(t)= & \boldsymbol{Y}^{-1}\left(t_{0}\right) \boldsymbol{k}(y)\left(t_{0}\right) \\
& +\int_{t_{0}}^{t} w(s)(f(s)+\lambda y(s))\left[\left(\theta_{1}, \cdots, \theta_{n}\right)(s) \cdot\right]^{*} d s
\end{aligned}
$$

for all $t$ in $(a, b)$. Since each of $\theta_{1}, \cdots, \theta_{n}, f$, and $y$ (by Theorem 2.1) is in $\mathscr{L}^{2}(w)$ it follows that the limits indicated exist, and that Eq. (2.2) will be satisfied if and only if

$$
\boldsymbol{k}(y)\left(t_{0}\right)=\boldsymbol{Y}\left(t_{0}\right)\left\{\boldsymbol{c}-\int_{t_{0}}^{a} w(s)(f(s)+\lambda y(s))\left[\left(\theta_{1}, \cdots\left(\theta_{n}\right)(s)\right]^{*} d s\right\} .\right.
$$

This is just a standard initial condition for solutions of Eq. (2.1); hence there is exactly one solution satisfying Eq. (2.3). The proof of the last assertion of the theorem is analogous. 
3. Maximal and minimal operators. For each operator $l$ and each compactifying weight $w, D$ denotes the set of all functions $y$ in $\mathscr{L}^{2}(w)$ which have (on each compact subinterval of $(a, b)$ ) an absolutely continuous $(n-1)$ st derivative and which have the property that $(1 / w) l(y)$ is in $\mathscr{L}^{2}(w) . \quad L$ (the maximal operator) denotes the restriction of $(1 / w) l$ to $D . D^{+}$and $L^{+}$are defined in the same may with $l$ replaced by $l^{+}$.

Let $\left(\varphi_{1}, \cdots, \varphi_{n}\right)$ be a linearly independent sequence of solutions to Eq. (1.2), and let $\boldsymbol{Y}=\boldsymbol{K}\left(\varphi_{1}, \cdots, \varphi_{n}\right) . \quad D_{0}$ denotes the set of all $y$ in $D$ satisfying

$$
\lim _{t \rightarrow a} \boldsymbol{Y}^{-1}(t) \boldsymbol{k}(y)(t)=0=\lim _{t \rightarrow b} \boldsymbol{Y}^{-1}(t) \boldsymbol{k}(y)(t) .
$$

Note that $D_{0}$ is independent of the fundamental system $\left(\varphi_{1}, \cdots, \varphi_{n}\right)$ which is used. (See Theorem 2.3 p. 70 of [1].) $L_{0}$ (the minimal operator) denotes the restriction of $L$ to $D_{0} . D_{0}^{+}$and $L_{0}^{+}$are defined in the same way with Eq. (1.2), $D$, and $L$ replaced respectively by Eq. (1.5), $D^{+}$, and $L^{+}$.

The main result of this section is presented in the following theorem. It is of interest to note that we are able, in the case of a compactifying weight, to deleniate the minimal operator through the boundary conditions (3.1); whereas in earlier treatments of similar problems, even with symmetric operators with maximal deficiency indices, (see $\S 17$ of [6] and $\S$ XIII. 2 of [3]). The minimal operator has been viewed less succinctly as the closure of what would correspond to the restriction of our operator $L$ to function with compact support interior to $(a, b)$. (See Corollary 3.5.)

THEOREM 3.1. Let $w$ be a compactifying weight for $l_{\text {. Then }} L_{0}$ is a densely defined operator on $\mathscr{L}^{2}(w)$,

$$
L_{0}^{*}=L^{+} \quad \text { and } \quad\left(L^{+}\right)^{*}=L_{0},
$$

where * denotes the adjoint operator in $\mathscr{L}^{2}(w)$.

The proof of this theorem will require the following lemmas, some of which were motivated by the material in $\S 17.3$ of [6].

LEMMA 3.2. Let $w$ be a compactifying weight for $l$ and let $f \in$ $\mathscr{L}^{2}(w)$. There is exactly one solution $y$ to

$$
l(y)=w f \text { a.e. on }(a, b)
$$

lying in $D_{0}$ if and only if $f$ is orthogonal to all solutions of $l^{+}(y)=0$ on $(a, b)$. Also $\mathscr{L}^{2}(w)$ is the orthogonal direct sum of range of $L_{0}$ and the null space of $L^{+}$. 
Proof. Using the notation of Theorem 2.2, let $y$ be the solution of Eq. (3.2) satisfying

$$
\lim _{t \rightarrow a} \boldsymbol{Y}^{-1}(t) \boldsymbol{k}(y)(t)=\mathbf{0} \text {. }
$$

By Theorem 2.1, $y$ is in $\mathscr{L}^{2}(w)$. Let $\lambda=0$, and $c=0$ in Eq. (2.3); multiplying both sides of this equation by $Y^{-1}\left(t_{0}\right)$, and taking the limit as $t_{0} \rightarrow b$ we see that $y$ will also satisfy

$$
\lim _{t \rightarrow b} \boldsymbol{Y}^{-1}(t) k(y)(t)=\mathbf{0},
$$

hence be in $D_{0}$, if and only if

$$
\mathbf{0}=\operatorname{column}\left(\left\langle f, \theta_{1}\right\rangle, \cdots,\left\langle f, \theta_{n}\right\rangle\right) \text {. }
$$

In view of Lemma 1.3 the first assertion is proved. Since the null space of $L^{+}$is of finite dimension, the Hilbert space $\mathscr{L}^{2}(w)$ is the orthogonal direct sum of it and its orthogonal complement. We have shown that this orthogonal complement is the range of $L_{0}$.

Lemma 1.4 and Theorem 2.2 allow us to give a particularly simple expression for Lagrange's identity. Note that if $w$ is a compactifying weight for $l$ then it is also a compactifying weight for $l^{+}$. Hence by Theorem 2.2 the vectors $z_{a}$ and $z_{b}$ defined below do exist.

LEMMA 3.3. Let $w$ be a compactifying weight for $l$. Let $\left(\varphi_{1}, \cdots\right.$, $\varphi_{n}$ ) be a linearly independent sequence of solutions to $E q$. (1.2) and let $\left(\theta_{1}, \cdots, \theta_{n}\right)$ be the adjoint of this sequence. For each $y \in D$ and $z \in D^{+}$let

$$
\boldsymbol{y}_{a}=\lim _{t \rightarrow a}\left[\boldsymbol{K}\left(\varphi_{1}, \cdots, \varphi_{n}\right)(t)\right]^{-1} \boldsymbol{k}(y)(t)
$$

and

$$
\boldsymbol{z}_{a}=\lim _{t \rightarrow a}\left[\boldsymbol{K}\left(\theta_{1}, \cdots, \theta_{n}\right)(t)\right]^{-1} \boldsymbol{k}(\boldsymbol{z})(t),
$$

and let $\boldsymbol{y}_{b}$ and $\boldsymbol{z}_{b}$ be defined in the same way taking the limits at $b$ rather than at $a$.

It follows that if $y \in D$ and $z \in D^{+}$then

$$
\langle L y, z\rangle-\left\langle y, L^{+} z\right\rangle=\boldsymbol{z}_{b}^{*} \boldsymbol{y}_{b}-\boldsymbol{z}_{a}^{*} \boldsymbol{y}_{a} .
$$

Proof. If $a<\alpha<\beta<b$ then

$$
\begin{aligned}
\int_{\alpha}^{\beta}\left(\frac{1}{w}\right) l(y) \bar{z} w-\int_{a}^{\beta}\left(\frac{1}{w}\right) \overline{l^{+}(z)} y w \\
=\int_{\alpha}^{\beta}\left[l(y) \bar{z}-y \overline{\left.l^{+}(z)\right]}\right. \\
=\left.\left\{[\boldsymbol{k}(z)]^{*} \boldsymbol{P k}(y)\right\}\right|_{\alpha} ^{\beta}
\end{aligned}
$$


where $P$ is the concomitant matrix for $l$. (See pp. 86 and 285 of [1].) In view of Lemma 1.4 this last expression is the same as

$$
\left.\left\{\left[\left[\boldsymbol{K}\left(\theta_{1}, \cdots, \theta_{n}\right)\right]^{-1} \boldsymbol{k}(z)\right]^{*}\left[\boldsymbol{K}\left(\varphi_{1}, \cdots, \varphi_{n}\right)\right]^{-1} \boldsymbol{k}(y)\right\}\right|_{\alpha} ^{\beta} \text {. }
$$

The conclusion to the lemma then follows by taking limits as $\beta \rightarrow b$ and $\alpha \rightarrow a$.

LEMMA 3.4. If the hypotheses of Lemma 3.3 are satisfied and each of $\boldsymbol{c}_{1}$ and $\boldsymbol{c}_{2}$ is in $\mathscr{C}^{n}$ then there is a $y \in D$ satisfying

$$
\boldsymbol{y}_{a}=\boldsymbol{c}_{1} \text { and } \boldsymbol{y}_{b}=\boldsymbol{c}_{2}
$$

and there is $a z \in D$ satisfying

$$
z_{a}=c_{1} \text { and } z_{b}=c_{2} .
$$

Proof. We shall show that there is a $u \in D$ such that $\boldsymbol{u}_{a}=\boldsymbol{c}_{1}$ and $\boldsymbol{u}_{b}=\mathbf{0}$. A similar argument would show that there is a $\boldsymbol{v} \in D$ such that $\boldsymbol{v}_{a}=0$ and $\boldsymbol{v}_{b}=\boldsymbol{c}_{2}$; then $y=u+v$ will satisfy the conclusion to the lemma.

Let $z_{j}$ be the solution to $l^{+}(y)=0$ on $(a, b)$ that

$$
z_{j a}=e_{i j}
$$

for $j=1,2, \cdots, n$ where $\boldsymbol{e}_{i j}$ is the $n \times 1$ matrix with $(i, j)$ entry 1 if $i=j$ and 0 otherwise. Since

$$
\left[\boldsymbol{K}\left(\theta_{1}, \cdots, \theta_{n}\right)\right]^{-1} \boldsymbol{K}\left(z_{1}, \cdots, \boldsymbol{z}_{n}\right)
$$

has the limit $I$ (the $n \times n$ identity matrix) at $a$, it follows that $\boldsymbol{K}\left(z_{1}, \cdots, z_{n}\right)$ is nonsingular at some (hence all points) point in $(a, b)$. Thus $z_{1}, \cdots, z_{n}$ are linearly independent and their Gram determinate (computed with respect to the inner product of $\mathscr{L}^{2}(w)$ ) is nonzero. In view of this fact we may let $f$ be the linear combination of $z_{1}, \cdots, z_{n}$ such that

$$
\operatorname{column}\left(\left\langle f, z_{1}\right\rangle, \cdots,\left\langle f, z_{n}\right\rangle\right)=-c_{1} .
$$

By Theorem 2.2 we may let $u$ be the element in $D$ such that $L u=f$ and $\boldsymbol{y}_{b}=0$. By Lemma 3.3 it follows that

$$
\left\langle f, z_{j}\right\rangle=\left\langle L u, z_{j}\right\rangle=\left\langle u, L^{+} z_{j}\right\rangle-z_{j a}^{*} \boldsymbol{u}_{a},
$$

and since $L^{+} z_{j}=0$ for $j=1,2, \cdots, n$ and $z_{j a}=e_{i j}$ we have that $\boldsymbol{u}_{a}=\boldsymbol{c}_{1}$. The argument for the existence of the $z \in D^{+}$is similar.

Proof of Theorem 3.1. That $D_{0}$ is dense in $\mathscr{L}^{2}(w)$ follows from the fact that $D_{0}$ contains all $n$ times continuously differentiable func- 
tions with compact support interior to $(a, b)$.

For the remainder of the proof we will adopt the notation of Lemma 3.3.

If $y \in D_{0}$ and $z \in D^{+}$then $\boldsymbol{y}_{a}=\mathbf{0}=\boldsymbol{y}_{b}$ hence by Lemma 3.3,

$$
\left\langle L_{0} y, z\right\rangle=\langle L y, z\rangle=\left\langle y, L^{+} z\right\rangle \text {. }
$$

Thus $L^{+} \subseteq L_{0}^{*}$.

Suppose that $g \in L_{0}^{*}$. Let $h=L_{0}^{*} g$ and let $z$ be any element of $D^{+}$satisfying $l^{+}(z)=w h$ a.e. on $(a, b)$. (See Theorem 2.1.) If $y \in D_{0}$ it follows from Lemma 3.3 that $\langle y, h\rangle=\left\langle y, L^{+} z\right\rangle=\left\langle L_{0} y, z\right\rangle$ and it follows from the definition of the adjoint operator that $\langle y, h\rangle=\langle y$, $\left.L_{0}^{*} g\right\rangle=\left\langle L_{0} y, g\right\rangle$. Hence $\left\langle L_{0} y, z-g\right\rangle=0$ for all $y \in D_{0}$. From Lemma 3.2 we have that $\mathscr{L}^{2}(w)$ is the orthogonal direct sum of the range of $L_{0}$ and the null space of $L^{+}$. Thus $z-g$ (after modification on a set of measure zero) is in the null space of $L^{+}$. In particular $z-g \in D^{+}$ and since $z \in D^{+}$it follows that $g \in D^{+}$. Since $L^{+} g=L^{+} z$ and $L^{+} z=$ $h=L_{0}^{*} g$ it follows that $L_{0}^{*} \subseteq L^{+}$. Hence the fact that $L_{0}^{*}=L^{+}$has been established.

From $L_{0}^{*}=L^{+}$we have that $L_{0}^{* *}=\left(L^{+}\right)^{*}$ and since $A \subseteq A^{* *}$ for any densely defined operator $A$ it follows that $L_{0} \subseteq\left(L^{+}\right)^{*}$.

Applying the part of Theorem 3.1 that has been proved with $l$ replaced by $l^{+}$we find that $\left(L_{0}^{+}\right)^{*}=L$. Since $L_{0}^{+} \subseteq L^{+}$implying $\left(L^{+}\right)^{*} \subseteq\left(L_{0}^{+}\right)^{*}$ it follows that $\left(L^{+}\right)^{*} \subseteq L$. Thus if $y \in\left(L^{+}\right)^{*}$ then $y \in$ $D$ and $\left(L^{+}\right)^{*} y=L y$; and if $z \in D^{+}$, by definition of adjoint, we have

$$
\left\langle y, L^{+} z\right\rangle=\left\langle\left(L^{+}\right)^{*} y, z\right\rangle
$$

or

$$
\left\langle y, L^{+} z\right\rangle=\langle L y, z\rangle \text {. }
$$

On the other hand, by Lemma 3.3 it follows that

$$
\langle L y, z\rangle=\left\langle y, L^{+} z\right\rangle+\boldsymbol{z}_{b}^{*} \boldsymbol{y}_{b}-\boldsymbol{z}_{a}^{*} \boldsymbol{y}_{a} .
$$

Thus $z_{b}^{*} \boldsymbol{y}_{b}-z_{a}^{*} \boldsymbol{y}_{a}=0$ for all $z \in D^{+}$. Since by Lemma 3.4 there is a $z \in D^{+}$such that $z_{a}$ and $z_{b}$ have any preassigned values it follows that $\boldsymbol{y}_{a}=\boldsymbol{y}_{b}=0$. Since we already have $y \in D$ and $\left(L^{+}\right)^{*} y=L y$ it follows that $y \in D_{0}$ and $\left(L^{+}\right)^{*} y=L_{0} y$. Thus $\left(L^{+}\right)^{*} \cong L_{0}$. This completes the proof of the fact that $\left(L^{+}\right)^{*}=L_{0}$.

CoRollary 3.5. The operator $L_{0}$ is closed in $\mathscr{L}^{2}(w)$.

Proof. The adjoint of any densely defined operator is closed and by Theorem 3.1, 


$$
L_{0}^{* *}=\left(L^{+}\right)^{*}=L_{0} \text {. }
$$

4. Intermediate operators and their adjoints. In this section we shall consider operators which lie between the maximal and minimal operators and their adjoints. We shall continue to use the notation developed in $\S 3$ and assume that all our operators are based on an $n$th order operator $l$ with a compactifying weight. Furthermore, all vectors $\boldsymbol{y}_{a}, \boldsymbol{y}_{b}, \boldsymbol{z}_{a}$, and $\boldsymbol{z}_{b}$ are to be formed using an arbitrary but fixed sequence $\left(\varphi_{1}, \cdots, \varphi_{n}\right)$ (of linearly independent solutions to $\left.l(y)=0\right)$ and its adjoint. (See Lemma 3.3.)

If each of $\boldsymbol{M}$ and $\boldsymbol{N}$ is in $\mathscr{C}^{n}$ and $\boldsymbol{B}$ is the $n \times 2 n$ matrix $(M: N)$ then $D_{B}$ will denote the set of all $y \in D$ such that

$$
\boldsymbol{M} \boldsymbol{y}_{a}+\boldsymbol{N} \boldsymbol{y}_{b}=\mathbf{0}
$$

and $D_{B}^{+}$will denote the set of all $z \in D^{+}$such that

$$
z_{a}^{*}=c^{*} \boldsymbol{M} \text { and } z_{b}^{*}=-\boldsymbol{c}^{*} \boldsymbol{N} \text { for some } \boldsymbol{c} \in \mathscr{C}^{n} \text {. }
$$

$L_{B}$ and $L_{B}^{+}$will denote the restrictions of $L$ and $L^{+}$to $D_{B}$ and $D_{B}^{+}$ respectively.

The following theorem shows that the boundary conditions 4.1 and 4.2 deleneate mutually adjoint operators in $\mathscr{L}^{2}(w)$.

THEOREM 4.1. If each of $\boldsymbol{M}$ and $\boldsymbol{N}$ is in $\mathscr{C}^{n}$ then $\left(L_{\boldsymbol{B}}\right)^{*}=L_{\boldsymbol{B}}^{+}$ and $\left(L_{B}^{+}\right)^{*}=L_{B}$.

Proof. By Lemma 3.3, if $y \in D_{B}$ and $z \in D_{B}^{+}$then

$$
\left\langle L_{B} y, z\right\rangle-\left\langle y, L_{B}^{+} z\right\rangle=z_{b}^{*} \boldsymbol{y}_{b}-z_{a}^{*} \boldsymbol{y}_{a},
$$

and from 4.1 and 4.2 it follows that the right hand side of this equation is zero. Thus $L_{B}^{+} \subseteq L_{B}^{*}$.

By its definition we have that $L_{0} \subseteq L_{B}$, hence $L_{B}^{*} \subseteq L_{0}^{*}$ so from Theorem 3.1 we have that $L_{B}^{*} \subseteq L^{+}$. Thus $L_{B}^{*} z=L^{+} z$ for all $z$ in the domain of $L_{B}$. Suppose now that $z$ is in the domain of $L_{B}^{*}$. Then, by definition of adjoint $\langle L y, z\rangle=\left\langle L_{B} y, z\right\rangle=\left\langle y, L_{B}^{*}, z\right\rangle=\left\langle y, L^{+} z\right\rangle$, for all $y$ in $D_{B}$. On the other hand, by Lemma 3.3 we have that

$$
\langle L y, z\rangle-\left\langle y, L^{+} z\right\rangle=z_{b}^{*} \boldsymbol{y}_{b}-z_{a}^{*} \boldsymbol{y}_{a} .
$$

Hence $z_{b}^{*} \boldsymbol{y}_{b}-z_{a}^{*} \boldsymbol{y}_{a}=0$ for all $y \in D_{B}$. Or the vector $\left[\begin{array}{r}z_{a} \\ -z_{b}\end{array}\right]$ is orthogonal in $\mathscr{C}^{2 n}$ (with respect to the standard inner product) to the subspace of all vectors $\left[\begin{array}{l}\boldsymbol{y}_{a} \\ \boldsymbol{y}_{b}\end{array}\right]$ such that $y \in D_{B}$. We denote this subspace by $V$. In view of Lemma $3.4 V$ is the set of all vectors $\left[\begin{array}{l}\boldsymbol{u} \\ \boldsymbol{v}\end{array}\right]$ such that 


$$
M u+N v=0 .
$$

Therefore, another way to view $V$ is that it is the orthogonal complement in $\mathscr{C}^{2 n}$ of the column space of $\left[\begin{array}{l}M^{*} \\ N^{*}\end{array}\right]$. Hence $\left[\begin{array}{r}z_{a} \\ -z_{b}\end{array}\right]$ must be in this column space or

$$
\left[\begin{array}{r}
z_{a} \\
-z_{b}
\end{array}\right]=\left[\begin{array}{l}
M^{*} \\
N^{*}
\end{array}\right] c \text { for some } c \in \mathscr{C}^{n}
$$

Thus condition 4.2 is satisfied and $z \in D_{B}^{+}$. We have shown then that $L_{B}^{*} z=L^{+} z$ for all $z$ in the domain of $L_{B}^{*}$ and that this domain is a subset of $D_{B}^{+}$. Thus $L_{B}^{*} \subseteq L_{B}^{+}$. This completes the proof of the first assertion of the theorem.

Again conditions 4.1 and 4.2 imply that the right hand side of equation 4.3 is zero when $y \in D_{B}$ and $z \in D_{B}^{+}$. Thus $L_{B} \subseteq\left(L_{B}^{+}\right)^{*}$. Also from its definition $L_{0}^{+} \subseteq L_{B}^{+}$, hence $\left(L_{B}^{+}\right)^{*} \subseteq\left(L_{0}^{+}\right)^{*}$; so by Theorem 3.1 applied to $l^{+}$we have that $\left(L_{B}^{+}\right)^{*} \subseteq L$. If $y$ is in the domain of $\left(L_{B}^{+}\right)^{*}$ then

$$
\langle L y, z\rangle-\left\langle\left(L_{B}^{+}\right)^{*} y, z\right\rangle=\left\langle y, L_{B}^{+} z\right\rangle=\left\langle y, L^{+} z\right\rangle
$$

for all $z \in D_{B}^{+}$and from Lemma 3.3

$$
\langle L y, z\rangle-\left\langle y, L^{+} z\right\rangle=z_{b}^{*} \boldsymbol{y}_{b}-z_{a}^{*} \boldsymbol{y}_{a} .
$$

Thus $\boldsymbol{z}_{b}^{*} \boldsymbol{y}_{b}-\boldsymbol{z}_{a}^{*} \boldsymbol{y}_{a}=0$ for all $\boldsymbol{z} \in D_{\boldsymbol{B}}^{+}$or the vector $\left[\begin{array}{l}\boldsymbol{y}_{a} \\ \boldsymbol{y}_{b}\end{array}\right]$ is orthogonal in $\mathscr{C}^{2 n}$ to the subspace of all vectors $\left[\begin{array}{r}z_{a} \\ -z_{b}\end{array}\right]$ such that $z \in D_{B}^{+}$. We denote this subspace by $W$. Again by Lemma 3.4 we conclude that $W$ is the set of all vectors $\left[\begin{array}{l}\boldsymbol{u} \\ \boldsymbol{v}\end{array}\right]$ such that

$$
u^{*}=c^{*} M \text { and } \boldsymbol{v}^{*}=c^{*} \boldsymbol{N}
$$

for some $\boldsymbol{c} \in \mathscr{C}^{n}$ or that $W$ is the column space of the matrix $\left[\begin{array}{l}\boldsymbol{M}^{*} \\ \boldsymbol{N}^{*}\end{array}\right]$. Since $\left[\begin{array}{l}\boldsymbol{y}_{a} \\ \boldsymbol{y}_{b}\end{array}\right]$ is orthogonal to $W$ we have that $\boldsymbol{M} \boldsymbol{y}_{a}+\boldsymbol{N} \boldsymbol{y}_{b}=\mathbf{0}$. Thus $y \in D_{B}$ and we have completed the argument that $\left(D_{B}^{+}\right)^{*} \subseteq D_{B}$, and from $\left(L_{B}^{+}\right)^{*} \subseteq L$ we have that $\left(L_{B}^{+}\right)^{*} \subseteq L_{B}$. Thus $\left(L_{B}^{+}\right)^{*}=L_{B}$.

The next theorem shows that boundary conditions of the type 4.2 can be expressed by conditions of the type 4.1 and conversely.

THEOREM 4.2. Suppose that $\boldsymbol{M}, \boldsymbol{N} \in \mathscr{M}^{n}$ and that $m$ is the column rank of $\left[\begin{array}{c}\boldsymbol{M}^{*} \\ -\boldsymbol{N}^{*}\end{array}\right]$. Let $\boldsymbol{D}$ be a $2 n \times(2 n-m)$ matrix whose columns form a basis in $\mathscr{C}^{2 n}$ for the orthogonal complement of the column space of $\left[\begin{array}{r}\boldsymbol{M}^{*} \\ -\boldsymbol{N}^{*}\end{array}\right]$ and let $\boldsymbol{P}$ and $\boldsymbol{Q}$ be the $n \times(2 n-m)$ matrices such 
that $\boldsymbol{D}=\left[\begin{array}{l}\boldsymbol{P} \\ \boldsymbol{Q}\end{array}\right]$. It follows that $z \in D^{+}$satisfies condition 4.2 if and only if

$$
\boldsymbol{P}^{*} \boldsymbol{z}_{a}+\boldsymbol{Q}^{*} \boldsymbol{z}_{b}=0,
$$

and it follows that $y \in D$ satisfies condition 4.1 if and only if

$$
\boldsymbol{y}_{a}^{*}=\boldsymbol{c}^{*} \boldsymbol{P}^{*} \text { and } \boldsymbol{y}_{b}^{*}=-\boldsymbol{c}^{*} \boldsymbol{Q}^{*} \text { for some } \boldsymbol{c} \in \mathscr{C}^{n} \text {. }
$$

Proof. $z \in D^{+}$satisfies 4.2 if and only if $\left[\begin{array}{c}z_{a} \\ z_{b}\end{array}\right]=\left[\begin{array}{c}M^{*} \\ -N^{*}\end{array}\right] c$ for some $c \in \mathscr{C}^{n}$. This holds if and only if $\left[\begin{array}{l}z_{a} \\ z_{b}\end{array}\right]$ is in the column space of $\left[\begin{array}{c}M^{*} \\ -N^{*}\end{array}\right]$ and this is equivalent to $\left[\begin{array}{l}z_{a} \\ z_{b}\end{array}\right]$ being orthogonal to the orthogonal to the orthogonal complement of the column space of $\left[\begin{array}{c}M^{*} \\ -N^{*}\end{array}\right]$. Eq. (4.4) is simply another way of stating that $\left[\begin{array}{l}z_{a} \\ z_{b}\end{array}\right]$ is in the orthogonal complement of the column space of $\boldsymbol{D}$. The argument for the second assertion of the theorem is similar.

5. Invertibility and Green's functions. In this section we give a necessary and sufficient condition for the operator $L_{B}$, defined in $\S 4$, to be invertible and show how the inverse operator, when it exists, may be expressed as an integral operator of the HilbertSchmidt type.

Theorem 5.1. Let $\boldsymbol{M}, \boldsymbol{N} \in \mathbb{C}^{n}$, let $\boldsymbol{B}=(\boldsymbol{M}: \boldsymbol{N})$, and let $L_{B}$ be as in $\S 4$. It follows that $L_{B}$ is invertible if and only if the matrix $\boldsymbol{M}+\boldsymbol{N}$ is nonsingular.

Proof. Since $L_{B}$ is linear it is invertible if and only if the only solution to $L_{B} y=0$ is the zero function. $L_{B} y=0$ if and only if $y$ satisfies the boundary condition 4.1 and $y$ is a linear combination of the same sequence of solution $\left(\varphi_{1}, \cdots, \varphi_{n}\right)$ used to construct $\boldsymbol{y}_{a}$ and $\boldsymbol{y}_{b}$. Thus $L_{B} y=0$ if and only if

$$
\begin{aligned}
& \boldsymbol{M} \lim _{t \rightarrow a}\left[\boldsymbol{K}\left(\varphi_{1}, \cdots, \varphi_{n}\right)(t)\right]^{-1}\left[\boldsymbol{K}\left(\varphi_{1}, \cdots, \varphi_{n}\right)(t)\right] \boldsymbol{c} \\
+ & \boldsymbol{N} \lim _{t \rightarrow b}\left[\boldsymbol{K}\left(\varphi_{1}, \cdots, \varphi_{n}\right)(t)\right]^{-1}\left[\boldsymbol{K}\left(\varphi_{1}, \cdots, \varphi_{n}\right)(t)\right] \boldsymbol{c}=0 \\
& \text { or } \quad(\boldsymbol{M}+\boldsymbol{N}) \boldsymbol{c}=0
\end{aligned}
$$

where $c$ is the vector in $\mathscr{C}^{n}$ such that

$$
y=\left(\varphi_{1}, \cdots, \varphi_{n}\right) \boldsymbol{c} .
$$

Since Eq. 5.1 is satisfied only for $\boldsymbol{c}=\mathbf{0}$ if and only if $\boldsymbol{M}+\boldsymbol{N}$ is nonsingular the theorem is proved. 
Theorem 5.2. Let $\boldsymbol{M}, \boldsymbol{N} \in \mathscr{M}^{n}$, let $\boldsymbol{B}=(\boldsymbol{M}: \boldsymbol{N})$, let $L_{\boldsymbol{B}}$ be as in $\S 4$, and suppose that $L_{B}$ is invertible. If $f \in \mathscr{L}^{2}(w)$ then $y \in D_{B}$ and $L_{B} y=f$ if and only if $y(t)=\int_{a}^{b} G(t, s) f(s) w(s) d s$ for all $t \in(a, b)$ where

$$
G(t, s)=\left\{\begin{array}{c}
{\left[\left(\varphi_{1}, \cdots, \varphi_{n}\right)(t)\right](\boldsymbol{M}+\boldsymbol{N})^{-1} \boldsymbol{M}\left[\left(\theta_{1}, \cdots, \theta_{n}\right)(s)\right]^{*}} \\
\text { for } a<s<t<b \\
-\left[\left(\varphi_{1}, \cdots, \Phi_{n}\right)(t)\right](\boldsymbol{M}+\boldsymbol{N})^{-1} \boldsymbol{N}\left[\left(\theta_{1}, \cdots, \theta_{n}\right)(s)\right]^{*} \\
\text { for } a<t<s<b
\end{array}\right.
$$

wherein $\left(\Phi_{1}, \cdots, \Phi_{n}\right)$ is a linearly independent sequence of solutions to $l(y)=0$ on $(a, b)$ and $\left(\theta_{1}, \cdots, \theta_{n}\right)$ is its adjoint.

Proof. $y \in D_{B}$ and $L_{B} y=f$ if and only if condition 4.1 holds and $l(y)=w f$ a.e. on $(a, b)$. By Lemma 1.2 we see that this last differential equation holds if and only if

$$
\begin{aligned}
& {\left[\boldsymbol{K}\left(\varphi_{1}, \cdots, \varphi_{n}\right)(\tau)\right]^{-1} \boldsymbol{k}(y)(\tau) } \\
= & \left\{\left[\boldsymbol{K}\left(\varphi_{1}, \cdots, \varphi_{n}\right)(t)\right]^{-1} \boldsymbol{k}(y)(t)\right. \\
+ & \left.\int_{t}^{\tau}\left[\left(\theta_{1}, \cdots, \theta_{n}\right)(s)\right]^{*} f(s) w(s) d s\right\}
\end{aligned}
$$

whenever $t, \tau \in(a, b)$. Using the fact that each $\theta_{k}$ and $f$ is in $\mathscr{L}^{2}(w)$ we may conclude that if $l(y)=w f$ a.e. on $(a, b)$ then

$$
\begin{aligned}
\boldsymbol{y}_{a}= & {\left[\boldsymbol{K}\left(\Phi_{1}, \cdots, \Phi_{n}\right)(t)\right]^{-1} \boldsymbol{k}(y)(t) } \\
& -\int_{a}^{t}\left[\left(\theta_{1}, \cdots, \theta_{n}\right)(s)\right]^{*} f(s) w(s) d s
\end{aligned}
$$

and

$$
\begin{aligned}
\boldsymbol{y}_{b}= & {\left[\boldsymbol{K}\left(\varphi_{1}, \cdots, \varphi_{n}\right)(t)\right]^{-1} \boldsymbol{k}(y)(t) } \\
& +\int_{t}^{b}\left[\left(\theta_{1}, \cdots, \theta_{n}\right)(s)\right]^{*} f(s) w(s) d s
\end{aligned}
$$

for all $t$ in $(a, b)$. Multiplying the first of these equations (on the left) by $\boldsymbol{M}$ and the second by $\boldsymbol{N}$ and adding we see that if $l(y)=f$ a.e. on $(a, b)$ and 4.1 is satisfied then

$$
\begin{aligned}
& (\boldsymbol{M}+\boldsymbol{N})\left[\boldsymbol{K}\left(\varphi_{1}, \cdots, \varphi_{n}\right)(t)\right]^{-1} \boldsymbol{k}(y)(t) \\
= & \boldsymbol{M} \int_{a}^{t}\left[\left(\theta_{1}, \cdots, \theta_{n}\right)(s)\right]^{*} f(s) w(s) d s \\
- & \boldsymbol{N} \int_{t}^{b}\left[\left(\theta_{1}, \cdots, \theta_{n}\right)(s)\right]^{*} f(s) w(s) d s .
\end{aligned}
$$

Using the fact that $M+N$ is nonsingular (see Theorem 5.1), solving Eq. (5.2) for $k(y)(t)$, and examining the first components of 
the resultant equation we see that the integral equation indicated in the theorem is satisfied.

If the integral equation in the theorem is satisfied then differentiating we find that

$$
\begin{aligned}
y^{\prime}(t)= & \sum_{k=1}^{n} \varphi_{k}(t) \overline{\theta_{k}(t)} f(t) w(t) \\
& +\int_{a}^{t}\left[\left(\varphi_{1}, \cdots, \varphi_{n}\right)^{\prime}(\boldsymbol{M}+\boldsymbol{N})^{-1} \boldsymbol{M}\left[\left(\theta_{1}, \cdots, \theta_{n}\right)\right]^{*} f(s) w(s) d s\right. \\
& -\int_{t}^{b}\left[\left(\varphi_{1}, \cdots, \varphi_{n}\right)^{\prime}(\boldsymbol{M}+\boldsymbol{N})^{-1} \boldsymbol{N}\left[\left(\theta_{1}, \cdots, \theta_{n}\right)\right]^{*} f(s) w(s) d s\right.
\end{aligned}
$$

for all $t$ in $(a, b)$. Returning to Definition 1.1 we see that $\sum_{k=1}^{n} \phi_{k}^{(j)} \bar{\theta}_{k}$ is the $(j+1, n)$ entry of the $n \times n$ identity matrix. In case $n=1$ Eq. (5.2) is immediate from the integral equation of the theorem, and in case $n \geqq 2$ the last observation and continued differentiation of Eq. (5.3) shows that Eq. (5.2) is satisfied. Taking the limits as $t \rightarrow a$ and as $t \rightarrow b$ in Eq. (5.2) we find that

$$
\boldsymbol{M} \boldsymbol{y}_{a}+\boldsymbol{N} \boldsymbol{y}_{b}=\left[-\boldsymbol{M}(\boldsymbol{M}+\boldsymbol{N})^{-1} \boldsymbol{N}+\boldsymbol{N}(\boldsymbol{M}+\boldsymbol{N})^{-1} \boldsymbol{M}\right] \mathscr{J}
$$

where

$$
\mathscr{J}=\int_{a}^{b}\left[\left(\theta_{1}, \cdots, \theta_{n}\right)(s)\right]^{*} f(s) w(s) d s,
$$

and adding and subtracting $\boldsymbol{M}(\boldsymbol{M}+\boldsymbol{N})^{-1} \boldsymbol{M}$ in the term in brackets on the right side of Eq. (5.4) we see that condition 4.1 is satisfied.

Returning to Eq. (5.2), if we add and subtract

$$
N \int_{a}^{t}\left[\left(\theta_{1}, \cdots, \theta_{n}\right)(s)\right]^{*} f(s) w(s) d s
$$

on the right hand side we find that

$$
\begin{aligned}
\boldsymbol{k}(y)(t)= & \boldsymbol{K}\left(\varphi_{1}, \cdots, \varphi_{n}\right)(t)\left[-(\boldsymbol{M}+\boldsymbol{N})^{-1} \boldsymbol{N} \mathscr{\mathcal { J }}\right. \\
& \left.+\int_{a}^{t}\left[\left(\theta_{1}, \cdots, \theta_{n}\right)(s)\right]^{*} f(s) w(s) d s\right]
\end{aligned}
$$

for all $t$ in $(a, b)$. Letting $t_{0}$ be a point in $(a, b)$ and adding and subtracting

$$
\int_{t_{0}}^{a}\left[\left(\theta_{1}, \cdots, \theta_{n}\right)(s)\right]^{*} f(s) w(s) d s
$$

in the term in brackets in the last equation we see that

$$
\boldsymbol{k}(y)(t)=\boldsymbol{K}\left(\varphi_{1}, \cdots, \varphi_{n}\right)(t)\left[\boldsymbol{c}+\int_{t_{0}}^{t}\left[\left(\varphi_{1}, \cdots, \varphi_{n}\right)(s)\right]^{*} f(s) w(s) d s\right]
$$


for all $t \in(a, b)$ where $c$ is a constant vector in $\mathscr{C}^{n}$. Thus by (a slight modification of) Lemma $1.2 y$ is a solution to $l(y)=w f$ a.e. on $(a, b)$. Using Theorem 2.1 we may now conclude that $y \in D_{B}$ and $L_{B} y=f$.

\section{REFERENCES}

1. E. A. Coddington and N. Levinson, Theory of Ordinary Differential Equations, McGraw-Hill, New York, 1965.

2. R. H. Cole, Theory of Ordinary Differential Equations, Appleton-Century-Crofts, New York, 1968.

3. N. Dunford and J. T. Schwartz, Linear Operators, Part II, Interscience-Wiley, New York, and London, 1963.

4. H. E. Gollwitzer, A note on a functional inequality, Proc. Amer. Math. Soc., 23 (1969), 642-647.

5. K. S. Miller, Linear Differential Equations in the Real Domain, Norton, New York, 1963.

6. M. A. Naimark, Linear Differential Operators, Part II, Frederick Ungar, New York, 1968.

7. P. W. Walker, Asympotics for a class of weighted eigenvalue problems, Pacific J. Math., 40 (1972), 501-510.

Received August 18, 1972.

Virginia Polytechnic Institute

AND

StATE University 


\section{PACIFIC JOURNAL OF MATHEMATICS}

\section{EDITORS}

RICHARD ARENS (Managing Editor) University of California

Los Angeles, California 90024

R. A. Beaumon'T

University of Washington Seattle, Washington 98105
J. Dugundj1*

Department of Mathematics University of Southern California Los Angeles, California 90007

D. Gilbarg and J. Milgram Stanford University Stanford, California 94305

\section{ASSOCIATE EDITORS}

E. F. BECKENBACH

B. H. NeUMANN

F. WOLF

K. YOSHIDA

\section{SUPPORTING INSTITUTIONS}

UNIVERSITY OF BRITISH COLUMBIA CALIFORNIA INSTITUTE OF TECHNOLOGY UNIVERSITY OF CALIFORNIA MONTANA STATE UNIVERSITY UNIVERSITY OF NEVADA NEW MEXICO STATE UNIVERSITY OREGON STATE UNIVERSITY UNIVERSITY OF OREGON OSAKA UNIVERSITY

\section{UNIVERSITY OF SOUTHERN CALIFORNIA STANFORD UNIVERSITY UNIVERSITY OF TOKYO UNIVERSITY OF UTAH WASHINGTON STATE UNIVERSITY UNIVERSITY OF WASHINGTON AMERICAN MATHEMATICAL SOCIETY NAVAL WEAPONS CENTER}

* C. R. DePrima California Institute of Technology, Pasadena, CA 91109, will replace J. Dugundji until August 1974. 


\section{Pacific Journal of Mathematics}

\section{Vol. 49, No. $1 \quad$ May, 1973}

A. Bigard, Free lattice-ordered modules ...........................

Richard Bolstein and Warren R. Wogen, Subnormal operators in strictly cyclic

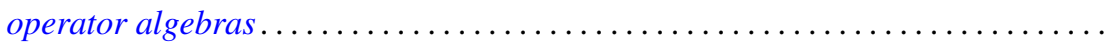

Herbert Busemann and Donald E. Glassco, II, Irreducible sums of simple

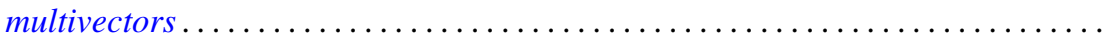

W. Wistar (William) Comfort and Victor Harold Saks, Countably compact groups

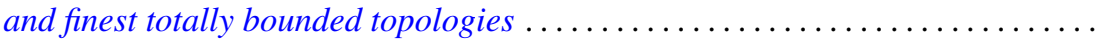

Mary Rodriguez Embry, Maximal invariant subspaces of strictly cyclic operator

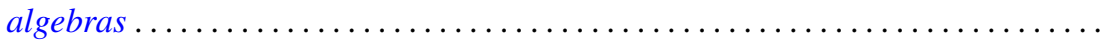

Ralph S. Freese and James Bryant Nation, Congruence lattices of semilattices......

Ervin Fried and George Grätzer, A nonassociative extension of the class of

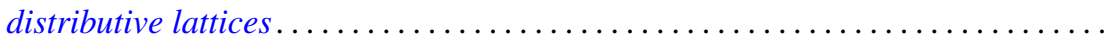

John R. Giles and Donald Otto Koehler, On numerical ranges of elements of locally

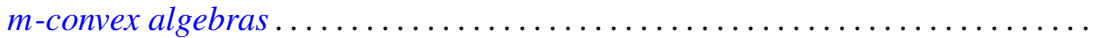

David A. Hill, On dominant and codominant dimension of $\mathrm{QF}-3$ rings ........ John Sollion Hsia and Robert Paul Johnson, Round and Pfister forms over $R(t) \ldots$ I. Martin (Irving) Isaacs, Equally partitioned groups . . . . . . . . . . . . . .

Athanassios G. Kartsatos and Edward Barry Saff, Hyperpolynomial approximation of solutions of nonlinear integro-differential equations.

Shin'ichi Kinoshita, On elementary ideals of $\theta$-curves in the 3-sphere and 2-links in the 4-sphere

Ronald Brian Kirk, Convergence of Baire measures

R. J. Knill, The Seifert and Van Kampen theorem via regular covering spaces ..

Amos A. Kovacs, Homomorphisms of matrix rings into matrix rings ..

Young K. Kwon, HD-minimal but no $H D$-minimal ..........

Makoto Maejima, On the renewal function when some of the mean renewal lifetimes are infinite

Juan José Martínez, Cohomological dimension of discrete modules over profinite groups.

W. K. Nicholson, Semiperfect rings with abelian group of units

Louis Jackson Ratliff, Jr., Three theorems on imbedded prime divisors of principal ideals.

Billy E. Rhoades and Albert Wilansky, Some commutants in $B(c)$ which are almost matrices

John Philip Riley Jr., Cross-sections of decompositions . . .

Keith Duncan Stroyan, A characterization of the Mackey uniformity $m\left(L^{\infty}, L^{1}\right)$ for

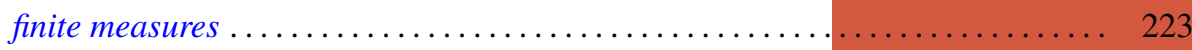

Edward G. Thurber, The Scholz-Brauer problem on addition chains . . . . . . . . . 229

Joze Vrabec, Submanifolds of acyclic 3-manifolds ............

Philip William Walker, Adjoint boundary value problems for compactified singular

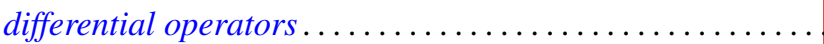

